Archives de sciences sociales des religions

196 | octobre-décembre 2021

Bulletin bibliographique

\title{
Nile GREEN, Global Islam. A Very Short Introduction
}

Oxford, Oxford University Press, 2020, 160 p.

Rachida Chih

\section{(2) OpenEdition}

1 Journals

\section{Édition électronique}

URL : https://journals.openedition.org/assr/64659

DOI : 10.4000/assr.64659

ISSN : $1777-5825$

Éditeur

Éditions de l'EHESS

\section{Édition imprimée}

Date de publication : 4 décembre 2021

Pagination : 283-286

ISBN : 9782713228735

ISSN : 0335-5985

Référence électronique

Rachida Chih, "Nile greEn, Global Islam. A Very Short Introduction », Archives de sciences sociales des religions [En ligne], 196 | octobre-décembre 2021, mis en ligne le 01 décembre 2021, consulté le 13 février 2022. URL : http://journals.openedition.org/assr/64659; DOI : https://doi.org/10.4000/assr. 64659

Ce document a été généré automatiquement le 13 février 2022.

(c) Archives de sciences sociales des religions 


\section{Nile GREEN, Global Islam. A Very Short Introduction}

Oxford, Oxford University Press, 2020, 160 p.

\section{Rachida Chih}

\section{RÉFÉRENCE}

Nile GREEN, Global Islam. A Very Short Introduction. Oxford, Oxford University Press, 2020, $160 \mathrm{p}$.

1 D'abord comme objet matériel, le livre est une réussite: Global Islam se présente, en effet, sous un petit format de belle finition, pratique à transporter et agréable à feuilleter. Il est publié par les presses universitaires d'Oxford dans une collection, Very Short Introduction Series, qui entend offrir une entrée en matière complète sur toutes sortes de sujets en faisant appel aux plus grands spécialistes dans leur domaine. Elle est donc destinée à un public large mais curieux et se veut accessible, tout en conservant une certaine érudition. Dans Global Islam, l'auteur fait, certes, un effort de clarté en multipliant les définitions des termes et des concepts, objet de rappels systématiques, et en faisant d'utiles récapitulatifs à chaque fin de chapitre. Il reste que le sujet tel qu'il est annoncé sur le rabat de la première de couverture est ambitieux et complexe : « The first comprehensive survey of the multiple versions of Islam propagated across geographical, political, and cultural boundaries during the era of modern globalisation. » L'ouvrage est un tour de force puisqu'il présente en 160 petites pages dont 139 de texte un tableau le plus complet possible des transformations et des mécanismes de diffusion de l'islam au cours d'une période qui s'étend de 1870 à nos jours ; la tâche est d'autant plus impressionnante que cette période de 150 années est celle d'une multiplication des redéfinitions, réinterprétations et réinventions de l'islam et de ses rituels, en compétition et en interaction entre elles, dans une pluralité de contextes géographiques, politiques et culturels, selon différentes temporalités. 
2 Nile Green est un historien britannique, professeur à UCLA, et un véritable chercheurglobe-trotter qui a voyagé dans un nombre incalculable de pays musulmans. Il a commencé par travailler sur le soufisme aux XVIII et $\mathrm{XIX}^{\mathrm{e}}$ siècles dans le sous-continent indien, une aire géographique où les musulmans ont toujours été confrontés à d'autres cultures religieuses, entre sunnites et chiites, entre musulmans et hindous puis missionnaires chrétiens arrivés dans le sillage de la colonisation britannique. Dans un ouvrage pionnier, Bombay Islam (2011), Green suit la trace des réseaux qui relient les musulmans entre l'Inde, l'Iran, l'Afghanistan, l'Océan indien et l'Afrique du Sud selon une approche méthodologique novatrice: il met à jour des pages méconnues de l'histoire intellectuelle de l'islam non pas à travers l'étude de sa tradition discursive mais au prisme de la métaphore du marché et de l'analyse de microportraits d'entrepreneurs religieux itinérants ; l'accent est mis sur les techniques, mécanismes et infrastructures que ces derniers adoptent pour faire la promotion de commodités religieuses dans ce marché hautement compétitif qu'est Bombay au xix siècle. Green applique, par la suite, cette approche à d'autres terrains d'échanges entre musulmans et non-musulmans, le Japon, l'Europe et l'Amérique, et s'impose comme un des meilleurs historiens des multiples formes de globalisation de l'islam grâce à ses nombreuses publications. Citons entre autres: Sufism, a Global History (Wiley Blackwell, 2012) ; avec James Gelvin, eds, Global Muslims in the Age of Steam and Print (University of California Press, 2013) ; Terrains of Exchange: Religious Economies of Global Islam (Oxford University Press, 2014).

3 Dans son introduction, l'auteur entend clarifier deux choses : 1) l'islam globalisé n'est en rien une version unique, homogène et dominante de l'islam mais se déploie, au contraire, en une grande variété de versions ; 2) les formes d'islamités contemporaines ont beaucoup à voir avec la modernité et la globalisation du monde moderne et peu avec une tradition islamique séculaire qui se serait fixée durant la période médiévale. L'auteur propose une chronologie de ces formes de globalisation en trois étapes qui correspondent aux trois chapitres suivants: Islam in the age of empire, steam and print; Defending Islam from the secular world order; From Islamic revolution to the Internet. Au cours de ces trois périodes, les années 1870-1920, 1920-1970, puis 1970 à nous jours, de nouveaux types d'activistes musulmans, d'organisations et des États indépendants utilisent les mécanismes de globalisation pour diffuser à l'échelle transnationale leur propre version de l'islam. Ces versions d'islam globalisé sont le résultat d'un processus historique auquel participent autant les musulmans que les non-musulmans.

Le premier chapitre, What is "global Islam"? développe la problématique énoncée en introduction et présente l'approche méthodologique adoptée. L'attention est moins portée sur les discours souvent stéréotypés sur l'islam que sur les trajectoires internationales des acteurs et des organisations qui font la promotion de ces nouvelles définitions et formes militantes d'appartenance et d'identification à l'islam. La globalisation de l'islam correspond à celle de sa réformation selon un processus qui se poursuit jusqu'à aujourd'hui ; de nouveaux acteurs religieux rejettent la culture et les institutions qui ont représenté l'islam pendant des siècles, celles des oulémas juristes et des soufis qui ont fini par faire bon ménage dès la fin de l'époque médiévale. L'auteur insiste sur cette rupture avec l'islam traditionnel ancré dans les lieux d'enseignement, de culte et de dévotion (madrasa, mosquées, couvents soufis) que les versions délocalisées de l'islam ont cherché à éradiquer comme autant de déviations du « pur et vrai islam », scripturaire et rationaliste, auquel ils prétendent éduquer les masses. Ces 
nouveaux acteurs (ou entrepreneurs) religieux adoptent des formes de prosélytisme et de militantisme empruntées aux modèles occidentaux, militent dans diverses associations, participent à des congrès, créent des partis, utilisent les nouveaux moyens de communication, de l'imprimerie aux chaînes câblées puis internet, et sont engagés dans une véritable compétition de légitimité autour de l'énonciation de la norme islamique.

Dans le second chapitre, Islam in the age of empire, steam and print, l'auteur est sur son terrain de prédilection: il montre comment l'impérialisme a créé les conditions politiques, économiques et technologiques de la transformation de l'islam au $\mathrm{xx}^{\mathrm{e}}$ siècle. La période entre 1870 et la Première Guerre mondiale a été identifiée par les historiens comme l'âge de la première globalisation ; jamais le monde n'a été aussi connecté grâce à la révolution des transports (bateau à vapeur, chemin de fer) et des communications (imprimerie, services postaux, télégraphe). L'auteur rappelle au passage que dans le monde musulman l'imprimerie privée ne se développe qu'à partir des années 1860 . Il souligne en outre un phénomène important au cours cette première phase : le net recul du rôle des États qui dans le passé en apportant leur patronage à des mosquées, madrasas et couvents soufis encourageaient une certaine vision de l'orthodoxie musulmane. Paradoxalement, en imposant sa domination, le pouvoir colonial a accordé une grande liberté religieuse (en particulier les Britanniques en Inde), créant ainsi un terrain fertile aux débats et échanges intellectuels. Ce chapitre dessine les portraits des premiers acteurs de la globalisation de l'islam, diplomates, étudiants, ou militants itinérants, d'origines diverses (iranien, indien, arabe, tatar), certains sont très connus comme al-Afghânî, mais d'autres le sont beaucoup moins. Leurs rencontres et échanges avec d'autres modèles d'entrepreneurs religieux, chrétiens, hindous ou nouveaux convertis à l'islam, ont lieu dans les hubs que sont les villes portuaires, Bombay, Beyrouth, Istanbul, Liverpool, Londres, mais aussi Paris, Le Caire et La Mecque. Ce chapitre est divisé en plusieurs thématiques dont les titres montrent la large étendue des champs d'investigation: The Ottoman campaign for Islamic Unity; The imperial networks of Islamic liberalism; Exporting Islamic reform from Russia; The making of Salafism; Itinerant Sufis of the Indian Ocean ; Globalizing Shi'ism ; Connecting southeast Asia and China ; India's messianic missionaries; Two failed political campaigns.

6 La période entre 1920 et 1970 (chapitre 3, Defending Islam from the secular world order) est celle de la désintégration des empires coloniaux, de l'essor des nationalismes et de la création d'États-nations sécularisés au moment des indépendances. Durant l'entredeux-guerres, ce sont des associations et des organisations à visée transnationale qui prennent le relais des actions jusqu'alors concentrées autour d'individus. Elles modèlent leurs formes de prosélytisme sur celles des évangélistes chrétiens en terre d'islam et investissent les domaines de l'éducation, de la santé et de la prédication. Mais au sortir de la colonisation, les nouveaux états indépendants reprennent le contrôle sur les activités religieuses qui sont soit instrumentalisées dans le cadre d'institutions étatiques, soit dénigrées et délégitimées dans le cas des confréries soufies, enfin, soit réprimées dans celui des associations militantes. Cependant, à l'aube des années 1970 l'échec des expériences socialistes des nouveaux États-nations sécularisés ouvre la voie de l'engagement politique aux organisations qui proposent l'islam comme la solution aux dilemmes politique, économique, éthique et spirituel de nombreux états musulmans dans des actions militantes, parfois violentes. Des états islamiques comme 
l'Arabie Saoudite, l'Iran, ou le Pakistan deviennent à leur tour des acteurs religieux globaux à travers leur politique étrangère.

7 Le dernier chapitre, From Islamic revolution to the Internet, analyse comme son titre l'indique, l'impact des profondes transformations géopolitiques, économiques et technologiques des cinquante dernières années : le jihâd en Afghanistan et la fin de la guerre froide, le triomphe du néolibéralisme et du libre-échange, la révolution iranienne, les nouveaux médias, de la cassette audio à internet, transforment les mécanismes de globalisation de l'islam. Ce chapitre retrace en particulier ce que Green a bien nommé la propre guerre froide de l'Arabie Saoudite, Saudia Arabia's own cold war, à partir du boum pétrolier des années 1970 (The oil boom of Saudi Salafism). L'islam global s'étend sur de nouveaux territoires avec les migrations de musulmans en Europe et en Amérique du Nord. La guerre idéologique menée par l'Arabie Saoudite prend un tournant, dont les conséquences ne sont pas mesurées à l'époque, avec le soutien financier et armé apporté aux leaders salafis de la résistance contre l'invasion soviétique en Afghanistan (The jihâd against godless socialism); ce faisant, elle porte le salafisme-jihadisme sur la scène internationale. Les multiples jihâds locaux qui en sont la conséquence directe sont passés en revue (The "little jihâds" of the 1990s) en particulier pour l'Afrique.

Global Islam fait le tour de la globalisation de l'islam sans sacrifier ni à sa complexité ni à sa diversité : toutes les étapes, tous les fronts et toutes les nouvelles frontières de l'expansion de l'islam globalisé sont reconnectées, toutes les stratégies de diffusion sont analysées selon les contextes mouvants de la géopolitique. Ce compte rendu donne juste un aperçu de ce tableau d'ensemble fouillé et en même temps très synthétique. Malgré le désormais vaste corpus d'études sur l'islam dans la globalisation, dont une sélection est donnée en bibliographie, il manquait cette vue d'ensemble, "the big picture ", et une chronologie des faits que Nile Green nous offre dans son livre. L'islam globalisé n'est pas un islam unique et unifié: l'ouvrage montre les divergences de visions théologiques et/ou d'agendas politiques, à travers les mini-portraits des acteurs qui ont construit le récit de cette globalisation. Le livre ne porte pas sur les doctrines qui finalement changent peu depuis que les premiers salafis en ont posé les bases mais sur les mécanismes de diffusion et les infrastructures de leur diffusion. L'islam globalisé est un islam salafisé selon un large éventail qui va du piétisme quiétiste à la violence jihadiste. L'auteur réitère en conclusion une mise garde énoncé dès son introduction : le livre porte sur l'action d'individus, d'organisations et d'États à l'échelle globale et si cette action touche en particulier des populations immigrées déracinées et coupées de leurs milieux culturels et religieux traditionnels, elle n'est pas représentative ou synonyme de ce à quoi tous les musulmans croient ou adhèrent. Ce vaste sujet qui dépasse le cadre de cet ouvrage nécessite un ensemble d'enquêtes sociologiques à grande échelle. Malgré les nombreuses études de cas qui porte aussi bien sur les diverses parties du monde musulman que sur la diaspora en Occident sur ce qu'être musulman aujourd'hui signifie, les implications de cette diversité ont encore du mal à être appréhendées par les historiens et sociologues de l'islam.

9 Ce petit ouvrage publié dans un format destiné au large public pourrait passer inaperçu du milieu des chercheurs sur l'islam et les sociétés musulmanes contemporaines; pourtant, il est d'une efficacité redoutable et, de mon point de vue, un outil de travail indispensable, car il offre cette nécessaire et indispensable mise en perspective de nos objets de recherche souvent très localisés et spécialisés. 